\title{
Design of crystal structures, morphologies and functionalities of titanium oxide using water-soluble complexes and molecular control agents
}

\author{
Makoto Kobayashi $^{1}$, Minoru Osada ${ }^{2}$, Hideki Kato ${ }^{1}$ and Masato Kakihana ${ }^{1}$ \\ Nature produces materials with excellent physical and chemical properties using water as a medium under ambient temperature \\ and pressure. The current need for more highly functional and compact materials makes the mimicking of natural processes to \\ produce these materials a very interesting approach, not only to obtain highly functionalized materials but also to reduce the \\ environmental impact. Our research group is engaged in the development of new stable water-soluble compounds that follow \\ natural processes for the synthesis of materials using water as a medium. This paper summarizes a hydrothermal synthesis \\ of rutile-type titanium oxide using water-soluble titanium complexes, controlling the crystal morphology by utilizing organic \\ molecules as shape-control agents as well as their speculative formation mechanism and superior dielectric properties of \\ unusually shaped titanium oxide crystals. By recapitulating our results, we indicate that control of the nano- and macro- \\ structures and functional improvement are accomplished through the development of new precursor compounds.
}

Polymer Journal (2015) 47, 78-83; doi:10.1038/pj.2014.89; published online 22 October 2014

\section{INTRODUCTION}

The affluence of modern society is supported by a diverse range of materials. To satisfy our increasing needs and desires, highly functional and more compact materials are needed. In general, there are two approaches to meet these needs: by developing new materials or improving existing materials. A good example of new material development is the discovery of iron-based superconductors. ${ }^{1,2}$ Iron-based materials were not originally thought to possess superconductivity; however, the discovery of the $\mathrm{La}\left[\mathrm{O}_{1-x} \mathrm{~F}_{x}\right] \mathrm{FeAs}$ superconductor drastically accelerated research into iron-based materials. One example of obtaining high functionality through the improvement of an existing material is the catalytic activity of gold nanoparticles. ${ }^{3,4}$ Gold has a high chemical stability and was assumed to be inactive, but the observation of a high catalytic activity for gold nanoparticles indicates that the functionality of existing materials can be improved by controlling their nano-/macro-structures. In addition, the discovery of high catalytic activity and hydrogen absorption properties for carbon nanotubes and nano-sheets (graphene) resulted from the development of a new material group as well as improvements to existing materials.

A variety of functional materials are present in the natural environment, many of which achieve amazingly high functionalization through hybridization of inorganic and organic materials. ${ }^{5-7}$ For example, pearl shells attain a high strength and a beautiful gloss from the combination of calcium carbonate and protein. Similarly, the crayfish's strong and soft shell is due to a combination of calcium carbonate and chitin. These are examples of materials in nature achieving a high functionality via the combination of simple components. By contrast, many organisms acquire excellent physical and chemical properties by forming peculiar shapes or controlling their crystal structure, which is difficult to achieve through chemical synthesis. ${ }^{8-12}$ As mentioned above, the functionality of materials in nature is significantly improved by combining different materials, forming complex shapes or controlling the nanostructure. These models were considered for the development of highly functionalized materials. Moreover, because natural processes proceed at ambient temperature and pressure, the ideal manufacturing techniques (that is, in environmentally benign processes) can be realized by learning from nature. Although these complex structures or shapes are formed under ambient temperature and pressure using water as the medium and DNA or other organics (mainly polymers) to control the shape, the details of many of these processes remain unknown. Therefore, many studies, including 'embryonic' studies, are required to establish processes that can imitate those found in nature.

Many of the chemical elements composing functional materials are water-insoluble, and even if an aqueous solution is obtained, it is stable only under a restricted circumstance (for example, $\mathrm{pH}$, atmosphere, temperature). Therefore, it is extremely difficult to achieve a new synthesis that mimics nature, with water as the medium and various organic molecules as shape-control agents, using existing raw

${ }^{1}$ Institute of Multidisciplinary Research for Advanced Materials, Tohoku University, Sendai, Japan and ${ }^{2}$ International Center for Materials Nanoarchitectonics (WPI-MANA), National Institute for Materials Science (NIMS), Ibaraki, Japan

Correspondence: Proferror M Kakihana, Institute of Multidisciplinary Research for Advanced Materials, Tohoku University, 2-1-1 Katahira, Aoba-ku, Sendai, Miyagi, 980-8577, Japan.

E-mail: kakihana@tagen.tohoku.ac.jp

Received 31 July 2014; revised 1 September 2014; accepted 2 September 2014; published online 22 October 2014 
materials. Titanium is a key element in material societies, and titanium salts, including titanium trichloride $\left(\mathrm{TiCl}_{3}\right)$, titanium tetrachloride $\left(\mathrm{TiCl}_{4}\right)$, titanium sulfate $\left(\mathrm{Ti}\left(\mathrm{SO}_{4}\right)_{2}\right)$ and titanium oxychloride $\left(\mathrm{TiOCl}_{2}\right)$, prepared from titanium chloride can be used as raw materials for aqueous solutions. These solutions are strongly acidic, and hydrous precipitates are formed through hydrolysis at a neutral $\mathrm{pH}$. Ammonium titanium oxalate $\left(\left(\mathrm{NH}_{4}\right)_{2} \mathrm{TiO}\left(\mathrm{C}_{2} \mathrm{O}_{4}\right)_{2}\right.$, abbreviated as $\left.\mathrm{TiO}(\mathrm{ox})_{2}\right)$ and ammonium hexafluorotitanate $\left(\left(\mathrm{NH}_{4}\right)_{2} \mathrm{TiF}_{6}\right.$, abbreviated as $\left.\mathrm{TiF}_{6}\right)$, among others, are commercially available and watersoluble compounds at a neutral $\mathrm{pH}$; however, they are highly toxic. In addition, solutions of $\mathrm{TiO}(\mathrm{ox})_{2}$ and $\mathrm{TiF}_{6}$ are not suitable for the synthesis of materials using various shape-control agents because they undergo hydrolysis under basic conditions. Titanium(IV) bis(ammonium lactato)dihydroxide is another option as a water-soluble titanium source. In fact, some research groups reported the synthesis of titanium compounds by a bio-inspired process employing titanium (IV) bis(ammonium lactato)dihydroxide and enzymes. ${ }^{13,14}$ However, there are problems regarding safety and handling because titanium(IV) bis(ammonium lactato)dihydroxide is composed of a flammable liquid and vapor. For these reasons, our first challenge was to develop a stable titanium solution to imitate the processes found in nature.

Our group has successfully developed many stable water-soluble compounds of water-insoluble elements, including titanium, ${ }^{15-22}$ tantalum $^{23}$ and niobium, ${ }^{24}$ which are key elements for functional materials. Recently, we focused on the development of water-soluble titanium complexes and discovered a series of new precursors that are safe $^{25}$ and stable at a wide range of $\mathrm{pH}$ values and do not precipitate even when mixed with various organic compounds. In addition, the high chemical stability and peculiar molecule structures of these titanium complexes resulted in the selective synthesis of titanium oxide polymorphs with a controlled shape by utilizing various additives such as carboxylic acids and amines. ${ }^{17,20,26-29}$ In this paper, we summarize our past studies on the synthesis and shape control of rutile-type titanium oxide using water-soluble titanium complexes with improved dielectric properties.

\section{GENERAL PROCEDURE FOR THE PREPARATION OF WATER-SOLUBLE TITANIUM COMPLEXES AND TITANIA}

The synthesis of water-soluble titanium complexes is described below. Titanium powder $(5 \mathrm{mmol})$ was dissolved in a solution of hydrogen peroxide $(30 \%, 20 \mathrm{ml})$ and ammonium hydroxide $(28 \%, 5 \mathrm{ml})$. The titanium powder dissolved immediately, and then a yellow peroxo titanium complex formed. After $2 \mathrm{~h}$, when the titanium metal had completely dissolved, a complexing agent was added. The amount of complexing agent depended on the compound used; that is, 1.5 equiv. of glycolic acids $(7.5 \mathrm{mmol})$ and 1.0 equiv. of citric acid ( $5 \mathrm{mmol}$ ) was added per mole of titanium. ${ }^{22}$ Excess hydrogen peroxide and ammonia were removed by heating at $353 \mathrm{~K}$ until the volume of the solution reduced by approximately half after the addition of the complexing agent to obtain an aqueous solution of the desired titanium complex as its ammonium salt. Aqueous solutions of complexes other than ammonium salts can also be produced by employing ion exchange resins or salts, such as sodium citrate rather than citric acid, as the complexing agent. ${ }^{30}$ The titanium complexes are stable under a broad range of solution $\mathrm{pH}$ levels and can be handled without hydrolysis in the presence of various additives. Titanium oxide particles were produced through hydrothermal treatment of a certain concentration of solutions of titanium complexes.

\section{SYNTHESIS AND MORPHOLOGY CONTROL OF RUTILE-TYPE TITANIUM OXIDE}

Among the titanium oxide polymorphs, rutile is the most stable phase thermodynamically, and anatase is easy to obtain from chemical synthesis. To obtain the desired crystalline phases of titanium oxide, control of the nucleation and phase transitions is vital. ${ }^{31-33}$ Polymorphic differences of titanium oxide can be explained by the differences in the packing of the $\mathrm{TiO}_{6}$ octahedra. Therefore, selective synthesis of single polymorphs of titanium dioxide can be accomplished by controlling the number of shared edges and vertexes, using octahedral $\mathrm{TiO}_{6}$ as the structure unit. However, packing the structural units as desired is extremely difficult. Nevertheless, many successful examples of the selective synthesis of both anatase and rutile

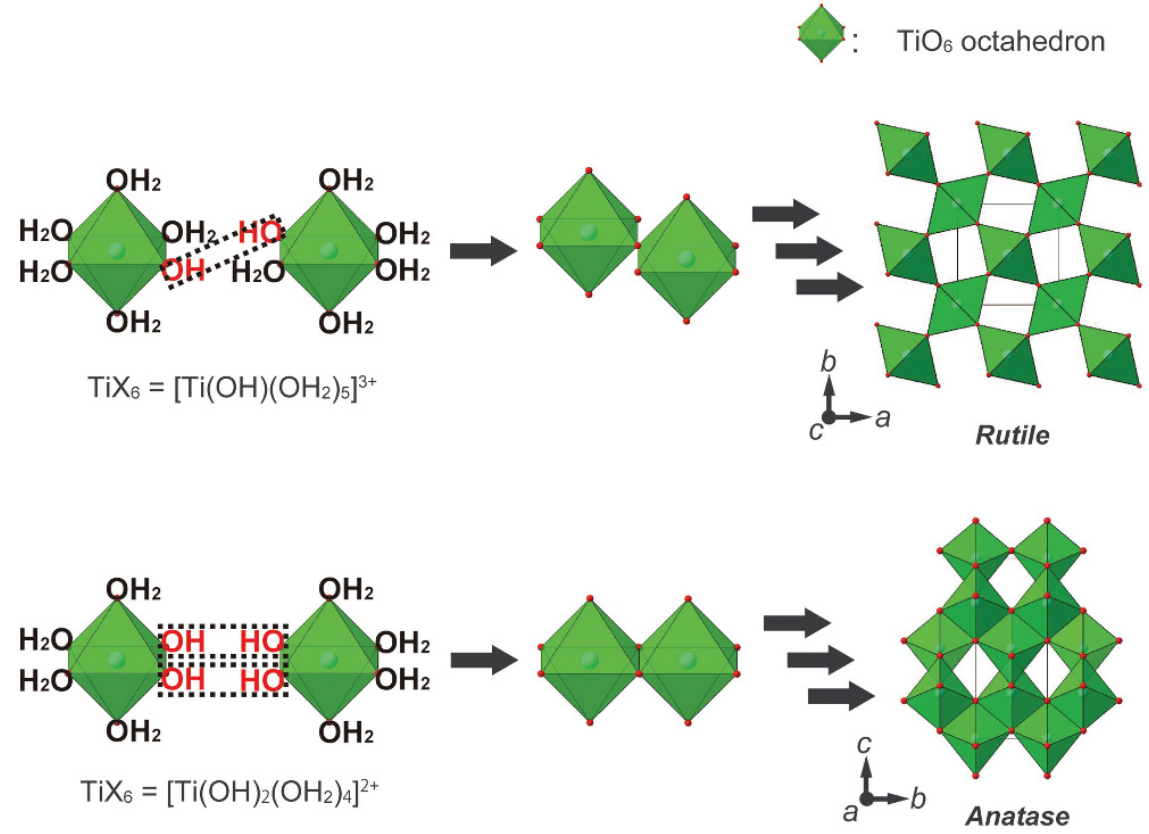

Figure 1 Schematic illustration of the formation mechanism of rutile (top) and anatase (bottom). 
polymorphs of titanium oxide have been reported. These materials were produced by accumulating titanium species $\left(\mathrm{TiX}_{6}: \mathrm{X}=\right.$ ligand) in solution while controlling the $\mathrm{pH}$ and choosing appropriate additives or ligands to form the titanium species. ${ }^{34-45}$ Figure 1 summarizes this approach, which assumes that the $\mathrm{OH}$ and $\mathrm{OH}_{2}$ groups are the only ligands of the titanium species in solution. In this mechanism, the formed polymorph is dependent on the number of shared edges and vertexes of $\mathrm{TiO}_{6}$ that are present, which determines the titanium oxide nuclei formed by condensation of the octahedral space group of $\left[\mathrm{Ti}(\mathrm{OH})_{n}\left(\mathrm{H}_{2} \mathrm{O}\right)_{6-n}\right]^{(4-n)+}$ in solution through hydrolysis (hydrolysis, oxolation and olation). For example, if the number of $\mathrm{OH}$ groups is large ( $n$ is a large value), then the opportunity to form shared edges increases because formation of Ti-O-Ti bonds by $\mathrm{OH}$ group condensation occurs more often. As a result, anatase nuclei having a four-shared-edge structure are formed and anatase powders are easily obtained. By contrast, when the value of $n$ is small, the opportunity to form $\mathrm{Ti}-\mathrm{O}-\mathrm{Ti}$ bonds through octahedral titanium condensation decreases and the formation of shared vertexes predominantly produces rutile nuclei with a two-shared-edge and two-shared-vertex structure. When titanium chloride, titanium sulfate or titanium alkoxide is used, the chloride $\left(\mathrm{Cl}^{-}\right)$, sulfate $\left(\mathrm{SO}_{4}{ }^{2-}\right)$ or methoxy groups $\left(\mathrm{CH}_{3} \mathrm{O}^{-}\right)$bound to titanium in solution lead to the formation of a titanium species different from the formula $\left[\mathrm{Ti}(\mathrm{OH})_{n}\left(\mathrm{H}_{2} \mathrm{O}\right)_{6-n}\right]^{(4-n)+}$. Therefore, some research groups thought that it was possible to control the 'dimer' or 'oligomer' (shared edge, shared vertex) binding formats of titanium octahedra by controlling their binding and hydrolysis in solution, allowing selective synthesis of titanium oxide polymorphs. ${ }^{35,38-40,42,44,45}$ However, because control of the chemical species in the solutions prepared using those titanium sources is not easy, precise control of the formation of the polymorphs has not been achieved. When stable water-soluble titanium complexes are used, the structure of the complex and its reactivity control the rates of hydrolysis and bonding. In other words, the development of the complexes themselves controls the formation of the $\mathrm{TiX}_{6}$ unit that allows the crystal structure to be controlled. Structures of the titanium glycolic acid complex and the titanium citric acid complex are shown in Figure 2. ${ }^{15,16}$ The structures of the two water-soluble titanium complexes are significantly different, and, based on our theory, it can be rationalized that the final polymorph produced can be predicted and varied depending on the complex used.

Figure 3 shows transmission electron microscopy images and electron diffraction patterns of particles obtained after hydrothermal treatment of $20 \mathrm{ml}$ of $0.25 \mathrm{~m}$ titanium glycolic acid and citric acid a

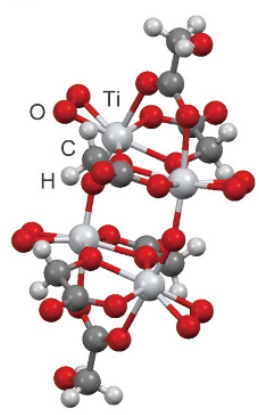

b

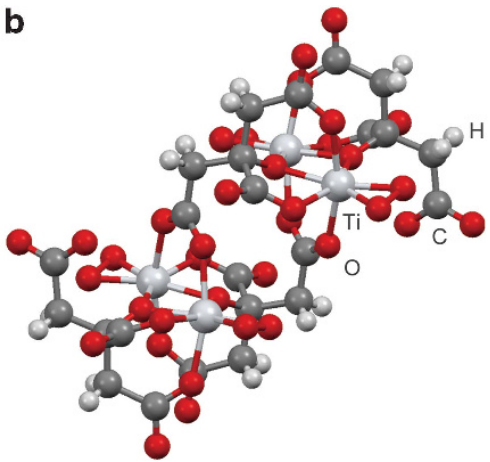

Figure 2 Structures of (a) an oxo-peroxo-glycolato titanium complex (titanium glycolic acid complex, $\left.\left[\mathrm{Ti}_{4}(\mathrm{ga})_{4}(\mathrm{Hga})_{2}(\mu-\mathrm{O})_{2}\left(\mathrm{O}_{2}\right)_{4}\right]^{6-}\right)^{15}$ and (b) a peroxo-citrato titanium complex (titanium citric acid complex, $\left.\left[\mathrm{Ti}_{4}\left(\mathrm{cit}^{*}\right)_{4}\left(\mathrm{O}_{2}\right)_{4}\right]^{8-}\right) \cdot{ }^{13}$ solutions at $473 \mathrm{~K}$ for $24 \mathrm{~h}$. Based on these images, powder X-ray diffraction data and Raman spectroscopic measurements, we concluded that the titanium glycolic acid complex produced rod-like rutile particles with dimensions of approximately $50 \times 150 \mathrm{~nm}$ and the titanium citric acid complex produced anatase crystals approximately $10 \mathrm{~nm}$ in size. ${ }^{17,27,46,47}$ Hydrothermal treatment of titanium glycolic acid solutions produced by-products of anatase nano-particles in the early stage of the reaction. These anatase particles disappeared as the hydrothermal treatment was prolonged, producing rutile as a single phase. ${ }^{46}$ In contrast, after hydrothermal treatment of titanium citric acid solutions, the rutile phase was not formed even when the reaction time was prolonged. ${ }^{27}$ By addition, rutile is not produced through the hydrothermal treatment of commercially available anatase-type titanium oxide particles in the presence of glycolic acid. ${ }^{46}$ Therefore, in the hydrothermal-assisted hydrolysis reaction of the titanium glycolic acid complex, rutile crystal can be obtained from rutile nuclei produced by the decomposition of the complex. By contrast, the titanium citric acid complex produces only anatase nuclei through a similar treatment. The difference in the polymorph produced is a result of the structural difference between the complexes because the structures of the titanium oxide nuclei composed of $\mathrm{TiO}_{6}$ are controlled by $\mathrm{X}$ in the complexes. Additional systematic experiments are needed to clarify the relationship between the complex structures and the crystal forms (polymorphs) of titanium oxide produced; however, it is possible to control the crystal structure at a nanomolecular level by developing a diverse range of precursor compounds. ${ }^{22}$

Figure 4 shows transmission electron microscopy images of particles obtained by hydrothermal treatment at $473 \mathrm{~K}$ for 3, 6, 24 and $100 \mathrm{~h}$ of titanium glycolic acid complex solutions $([\mathrm{Ti}]=0.25 \mathrm{M}, 20 \mathrm{ml})$ with an excess of glycolic acid ( $15 \mathrm{mmol}, 3$ times to Ti) as an additive. The $\mathrm{X}$-ray diffraction measurement confirmed that hydrothermal treatment for $24 \mathrm{~h}$ or less provided a multi-phase of anatase and rutile, whereas treatment for $100 \mathrm{~h}$ provided a single-phase of rutile. ${ }^{46,48}$ Comparison of the rutile particles obtained with no additive (Figure 3a) to those with additive indicated that the additional glycolic acid promoted growth along the $c$-axis of the rod-like rutile crystals, thereby increasing the aspect ratio. As the reaction time increased, a decrease in the ratio of fine particles was accompanied by the significant growth of rod-like rutile particles. This observation indicates that Ostwald ripening ${ }^{49}$ of the rod-like rutile particles occurs by dissolving anatase nano-particles and rutile particles. The yield of each reaction increased for longer reaction times: less than $1 \%$ for $3 \mathrm{~h}$, $1.0 \%$ for $6 \mathrm{~h}, 2.8 \%$ for $24 \mathrm{~h}$ and $40.5 \%$ for $100 \mathrm{~h}$. Accordingly, nuclei formation and crystal growth are thought to occur simultaneously in this reaction system.

Anisotropic growth in the direction of the $c$-axis, mentioned previously, is a result of crystal growth control by unique absorption of glycolic acids to $\{110\} .{ }^{46,48}$ Similar anisotropic growth along the $c$-axis was observed when titanium chloride was used instead of titanium complexes, indicating that the anisotropic growth is not dependent on the titanium source used. When lactic acid or hydroxybutyric acid was used, the particles produced had similar anisotropic growth along the $c$-axis. By contrast, when oxalic acid was the additive and the $\mathrm{pH}$ was adjusted to approximately 3 , the same $\mathrm{pH}$ value as in the glycolic acid conditions, rod-like rutile particles were formed with an aspect ratio of only 1.5. ${ }^{46}$ The reason for the low aspect ratio is the fact that oxalic acid has two carboxyl groups that can strongly bind to all of the exposed faces of rutile crystals, thus not stabilizing a specific crystal face. ${ }^{50}$ By contrast, glycolic acid, lactic acid and hydroxybutyric acid are monocarboxylic acids, and their 

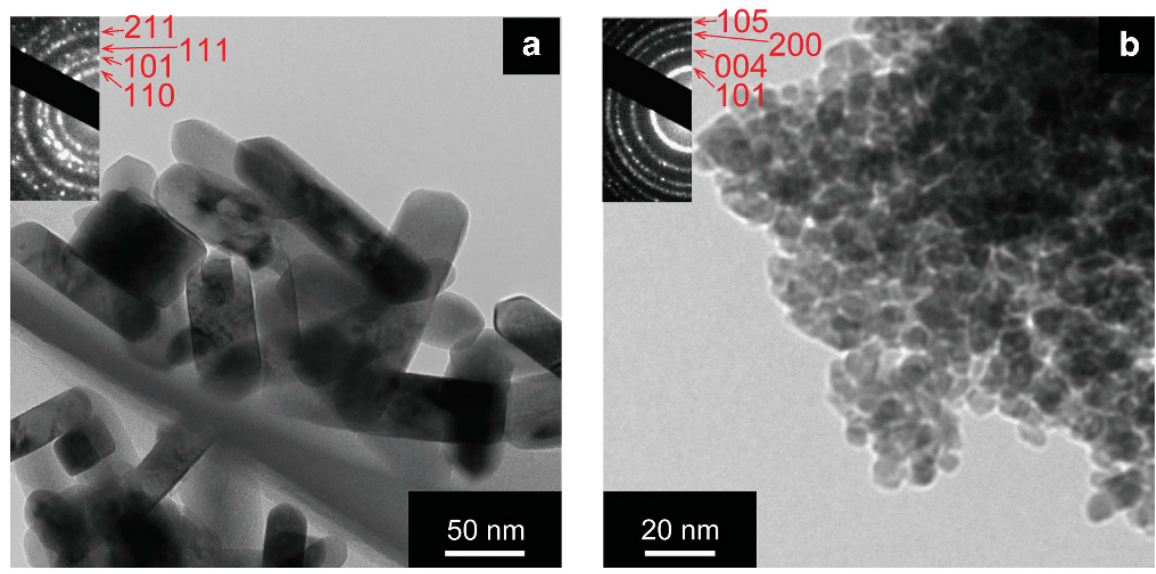

Figure 3 Transmission electron microscopy images of powders synthesized by hydrothermal treatment of aqueous solutions containing (a) a titanium glycolic acid complex and (b) a titanium citric acid complex at $473 \mathrm{~K}$ for $24 \mathrm{~h}([\mathrm{Ti}]=0.25 \mathrm{M})$.
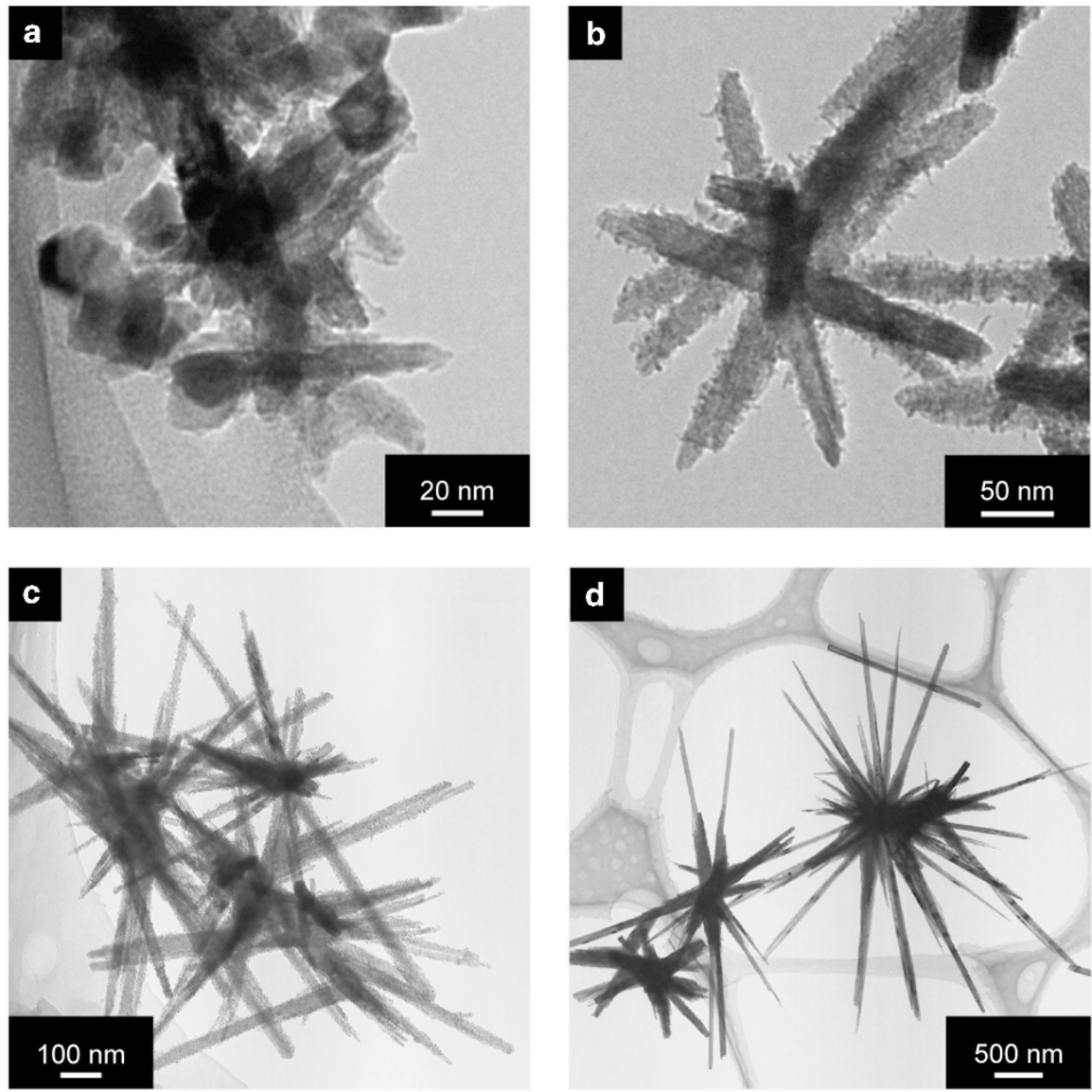

Figure 4 Transmission electron microscopy images of powders synthesized by hydrothermal treatment of titanium glycolic acid complex aqueous solutions in the presence of glycolic acid as an additive at $473 \mathrm{~K}$ for (a) 3, (b) 6 , (c) 24 and (d) $100 \mathrm{~h}$ ([Ti] $=0.25 \mathrm{~m}$, [additive]/[Ti] = 3).

adsorption on a specific crystal face results in the promotion of anisotropic growth. A similar hydrothermal treatment after adding glycolic acids and adjusting the $\mathrm{pH}$ to 8 , which exceeds the isoelectric point of titanium oxide $(4.7-6.7)$, provides rutile particles similar to the rod-like particles obtained in the absence of glycolic acid. ${ }^{46}$ Glycolic acid dissociates in solution to carboxylate anions, which enable unique absorption to complementary titanium dangling bonds on $\{110\}$. We estimated that the crystals formed were negatively charged in the regions and the adsorption of glycolic acids on a specific crystal face was inefficient. Therefore, no change in surface energy occurred, resulting in the failure to control the crystal growth. There is a tendency to produce particles with high anisotropy when hydroxybutyric acid is used in place of glycolic acid as an additive, which indicates that rutile crystals with higher anisotropy might be synthesized using hydroxy-carboxylic acids with larger alkyl groups as control agents. To design such anisotropic growth more precisely, the combination of calculation and simulation with experimental results is a powerful approach. ${ }^{51}$ 


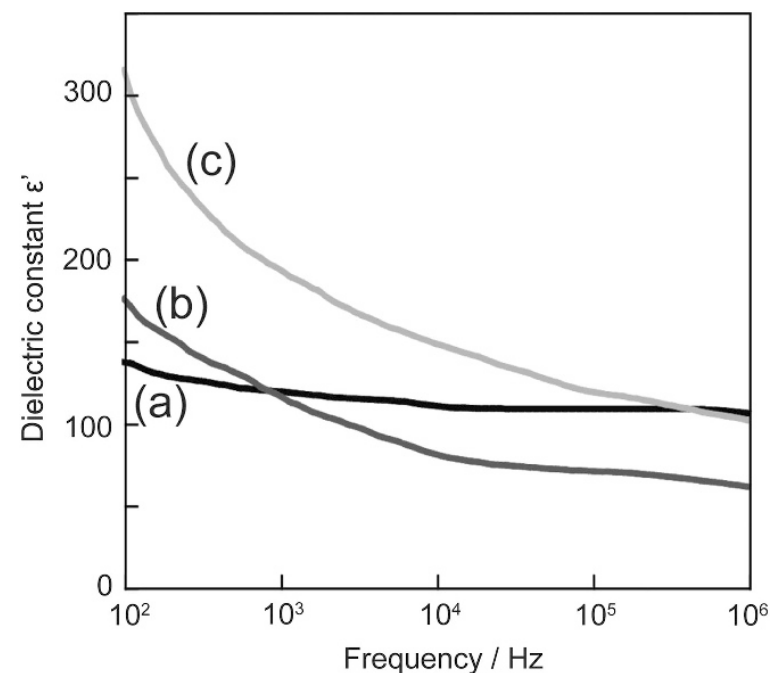

Figure 5 Dielectric permittivity of (a) bulk (approximately $1 \mu \mathrm{m}$ ) rutile powder, (b) rod- and (c) whisker-like (high aspect ratio) rutile crystals synthesized using a water-soluble titanium glycolic acid complex. A full color version of this figure is available at Polymer Journal online.

\section{DESIGN OF HIGH-K TITANIUM OXIDE}

The performance of titanium oxide is strongly influenced by its crystal structure and is therefore dependent on the polymorph chosen. The dielectric properties of the different polymorphs of titanium oxide vary considerably. Anatase has a dielectric constant $\left(\varepsilon_{\mathrm{r}}\right)$ of approximately 30 , whereas rutile has a $\varepsilon_{\mathrm{r}}$ of 120 ; the highest value among mono metal oxide-based systems. ${ }^{52-54}$ Dielectric properties are strongly dependent on crystal polarization and thus influenced by particle size, microstructure and grain boundary. ${ }^{55}$ The dielectric constant of rutile-type titanium oxide, which also depends on crystal orientation, is 180 in the direction of the $c$-axis and 90 in the direction of the $a$-axis. ${ }^{54,56}$ Therefore, synthesizing titanium oxide crystals with a high degree of anisotropy achieves a high functionality for this material as a dielectric compound. In addition, a broader understanding of highly functional materials can be achieved through the synthesis of these materials and the study of their properties.

Figure 5 shows the dielectric properties of rod-like rutile-type titanium oxide with an average dimension of $49 \times 136 \mathrm{~nm}$, whiskerlike rutile-type titanium oxide with an average dimension of $49 \times 1186 \mathrm{~nm}$, and a commercially available bulk rutile-type titanium oxide with a particle size larger than $1 \mu \mathrm{m} .{ }^{57}$ The dielectric properties were evaluated after kneading the titanium oxide powders with poly (vinyl butyral) and sintering the produced pellets at $723 \mathrm{~K}$. The dielectric property of commercial rutile particles was almost constant at 120 , regardless of the frequency used. By contrast, the dielectric constant of the rod-like rutile nanoparticles was approximately 170 at $100 \mathrm{~Hz}$, which was higher than the commercial product despite the nano-size of the particles. However, the dielectric constant decreased with increasing frequency. As a result, at $1 \mathrm{kHz}$, the constant was approximately 110 , lower than the commercial sample. ${ }^{58}$ By contrast, particles with larger aspect ratios showed a dielectric constant of approximately 300 at $100 \mathrm{~Hz}$, and the constant remained higher than that of the commercial product as the frequency increased to $100 \mathrm{kHz}$. The high crystallinity of the titanium oxides obtained through this approach is one of the reasons for their good dielectric properties. In addition, changes in the local structure produced by anisotropic growth is another major factor. The Raman spectra of the two synthesized samples (rod-like rutile and anisotropically grown rutile) showed a red shift in the $E_{\mathrm{g}}$ and $A_{1 \mathrm{~g}}$ modes compared with the commercial rutile particles. This shift is caused by softening of the in-plane Ti-O bond in titanium oxide crystals. ${ }^{57}$ Further support from computational modeling and various other fields, such as physics, is required to determine the exact cause of the large dielectric constant. We propose that the increase in polarization of [001], due to a softening of the in-plane Ti-O bond, results in the very large dielectric constants for rutile particles with larger aspect ratios and the equivalent constants for rod-like particles (nano-size) compared with commercial products (micron size). ${ }^{59}$

\section{SUMMARY}

In this paper, we describe a method to achieve concurrent control of both the nano-space of the crystal structure and the macro-space of the crystal shape for titanium oxide, a representative inorganic material. Development of precursors with unprecedented stability enabled the synthesis under a wide range of reaction conditions and the utilization of various shape-controlling agents. As a result, we could obtain anisotropically grown crystals with the use of simple compounds as shape-controlling agents. Moreover, by developing various precursors, we successfully demonstrated that the crystal structure of $\mathrm{TiO}_{2}$ and the three-dimensional arrangement of $\mathrm{Ti}$ and $\mathrm{O}$ on the atomic level were controllable. Conventionally, highly functional inorganic materials were obtained through post-treatments, such as a second heat treatment. By contrast, we believe that our suggestion that the microstructure can be controlled by the choice of precursors, which leads to a great improvement in the functionalities of inorganic materials, will have a significant impact on the material chemistry field. Synthesis of natural materials uses polymers instead of small molecules, such as those used in this study, and is achieved under milder conditions. We will continue our research into imitating nature to achieve 'fusion materials' with properties that surpass those found in natural materials.

\section{ACKNOWLEDGEMENTS}

We thank Dr V. Petrykin (SuperOX Japan) and Dr K. Tomita (Tokai Univ.) for fruitful discussions. This work was supported in part by a Grant-in-Aid for Scientific Research (no. 22107002) on the Innovative Areas: 'Fusion Materials' (area no. 2206) from the Japanese Government's Ministry of Education, Culture, Sports, Science and Technology (MEXT).

1 Kamihara, Y., Watanabe, T., Hirano, M., Hosono, H. Iron-based layered superconductor $\mathrm{La}\left[\mathrm{O}_{1-\mathrm{x}} \mathrm{F}_{\mathrm{x}}\right] \mathrm{FeAs}(\mathrm{x}=0.05-0.12)$ with $\mathrm{T}_{\mathrm{c}}=26 \mathrm{~K}$. J. Am. Chem. Soc. 130, 3296-3297 (2008)

2 Takahashi, H., Igawa, K., Arii, K., Kamihara, Y., Hirano, M., Hosono, H. Superconductivity at $43 \mathrm{~K}$ in an iron-based layered compound $\mathrm{LaO}_{1-\mathrm{x}} \mathrm{F}_{\mathrm{x}} \mathrm{FeAs}$. Nature 457, 376-378 (2008)

3 Haruta, M., Kobayashi, T., Sano, H., Yamada, N. Novel gold catalysts for the oxidation of carbon monoxide at a temperature far below $0^{\circ} \mathrm{C}$. Chem. Lett. 16, 405-408 (1987).

4 Haruta, M. Size- and support-dependency in the catalysis of gold. Catal. Today 36 153-166 (1997).

5 Kato, T., Sakamoto, T., Nishimura, T. Macromolecular templating for the formation of inorganic-organic hybrid structures. MRS Bull. 35, 127-132 (2010).

6 Aizenberg, J., Hendler, G. Designing efficient microlens arrays: lessons from Nature. J. Mater. Chem. 14, 2066-2072 (2004).

7 Aizenberg, J., Sundar, V. C., Yablon, A. D., Weaver, J. C., Chen, G. Biological glass fibers: Correlation between optical and structural properties. Proc. Natl Acad. Sci. USA 101, 3358-3363 (2004).

8 Kröger, N. Prescribing diatom morphology: toward genetic engineering of biological nanomaterials. Curr. Opin. Chem. Biol. 11, 662-669 (2007).

9 Imai, H., Oaki, Y. Emergence of helical morphologies with crystals: twisted growth under diffusion-limited conditions and chirality control with molecular recognition. CrystEngComm 12, 1679-1687 (2010).

10 Beniash, E., Aizenberg, J., Addadi, L., Weiner, S. Amorphous calcium carbonate transforms into calcite during sea urchin larval spicule growth. Proc. R. Soc. B 264 461-465 (1997). 
11 Weiss, I. M., Tuross, N., Addadi, L., Weiner, S. Mollusc larval shell formation: amorphous calcium carbonate is a precursor phase for aragonite. J. Exp. Zool. 293, 478-491 (2002).

12 Politi, Y., Levi-Kalisman, Y., Raz, S., Wilt, F., Addadi, L., Weiner, S., Sagi, I. Structural characterization of the transient amorphous calcium carbonate precursor phase in sea urchin embryos. Adv. Funct. Mater. 16, 1289-1298 (2006).

13 Luckarift, H. R., Dickerson, M. B., Sandhage, K. H., Spain, J. C. Rapid, roomtemperature synthesis of antibacterial bionanocomposites of lysozyme with amorphous silica or titania. small 2, 640-643 (2006).

14 Johnson, J. M., Kinsinger, N., Sun, C., Li, D., Kisailus, D. Urease-mediated roomtemperature synthesis of nanocrystalline titanium dioxide. J. Am. Chem. Soc. 134, 13974-13977 (2011).

15 Kakihana, M., Tada, M., Shiro, M., Petrykin, V., Osada, M., Nakamura, Y. Structure and Stability of Water Soluble $\left(\mathrm{NH}_{4}\right)_{8}\left[\mathrm{Ti}_{4}\left(\mathrm{C}_{6} \mathrm{H}_{4} \mathrm{O}_{7}\right)_{4}\left(\mathrm{O}_{2}\right)_{4}\right] \bullet 8 \mathrm{H}_{2} \mathrm{O}$. Inorg. Chem. 40 891-894 (2001)

16 Kakihana, M., Tomita, K., Petrykin, V., Tada, M., Sasaki, S., Nakamura, Y. Chelating of titanium by lactic acid in the water-soluble diammonium tris(2-hydroxypropionato) titanate(IV). Inorg. Chem. 43, 4546-4548 (2004).

17 Tomita, K., Petrykin, V., Kobayashi, M., Shiro, M., Yoshimura, M., Kakihana, M. A water-soluble titanium complex for the selective synthesis of nanocrystalline brookite, rutile, and anatase by a hydrothermal method. Angew. Chem. Int. Ed. 45, 2378-2381 (2006).

18 Kobayashi, M., Petrykin, V., Tomita, K., Kakihana, M. New water-soluble complexes of titanium with amino acids and their application for synthesis of $\mathrm{TiO}_{2}$ nanoparticles. J. Ceram. Soc. Jpn 116, 578-583 (2008).

19 Morishima, Y., Kobayashi, M., Petrykin, V., Yin, S., Sato, T., Kakihana, M., Tomita, K. Hydrothermal synthesis of brookite type $\mathrm{TiO}_{2}$ photocatalysts using a water-soluble Ticomplex coordinated by ethylenediaminetetraacetic acid. J. Ceram. Soc. Jpn 117 , 320-326 (2009).

20 Kakihana, M., Kobayashi, M., Tomita, K., Petrykin, V. Application of water-soluble titanium complexes as precursors for synthesis of titanium-containing oxides via aqueous solution processes. Bull. Chem. Soc. Jpn 83, 1285-1308 (2010).

21 Truong, Q. D., Kobayashi, M., Kato, H., Kakihana, M. Hydrothermal synthesis of hierarchical $\mathrm{TiO}_{2}$ microspheres using a novel titanium complex coordinated by picolinic acid. J. Ceram. Soc. Jpn 119, 513-516 (2011).

22 Yoshizawa, M., Kobayashi, M., Petrykin, V., Kato, H., Kakihana, M. Insights into a selective synthesis of anatase, rutile and brookite-type titanium dioxides by a hydrothermal treatment of titanium complexes. J. Mater. Res. 29, 90-97 (2014).

23 Petrykin, V., Kakihana, M., Yoshioka, K., Sasaki, S., Ueda, Y., Tomita, K., Nakamura, Y., Shiro, M., Kudo, A. Synthesis and structure of new water-soluble and stable tantalum compound: Ammonium tetralactatodiperoxo- $\mu$-oxo-ditantalate(V). Inorg. Chem. 45, 9251-9256 (2006).

24 Dey, D., Petrykin, V., Sasaki, S., Kakihana, M. Water soluble $\mathrm{Na}\left[\mathrm{Nb}\left(\mathrm{O}_{2}\right)_{3}\right]_{2} \bullet \mathrm{H}_{2} \mathrm{O}$ as a new molecular precursor for synthesis of sodium niobate. J Ceram. Soc. Jpn 115 , 808-812 (2007)

25 Nakasuji, K., Usuda, K., Kawasaki, T., Dote, E., Hayashi, S., Mitsui, G., Adachi, K., Fujihara, M., Shimbo, Y., Kono, K. Urinary and serum titanium-Assessment as an indicator of exposure to ammonium citratoperoxotitanate(IV) and its influence on renal function. Biol. Trace Elem. Res. 110, 119-131 (2006).

26 Kobayashi, M., Petrykin, V., Tomita, K., Yoshimura, M., Kakihana, M. One-step synthesis of $\mathrm{TiO}_{2}(\mathrm{~B})$ nanoparticles from a water-soluble titanium complex. Chem. Mater. 19, 5373-5376 (2007).

27 Tomita, K., Kobayashi, M., Petrykin, V., Yin, S., Sato, T., Yoshimura, M., Kakihana, M. Hydrothermal synthesis of $\mathrm{TiO}_{2}$ nano-particles using novel water-soluble titanium complex. J. Mater. Sci. 43, 2217-2221 (2008).

28 Kobayashi, M., Tomita, K., Petrykin, V., Yoshimura, M., Kakihana, M. Direct synthesis of brookite-type titanium oxide by hydrothermal method using water-soluble titanium complexes. J. Mater. Sci. 43, 2158-2162 (2008).

29 Kobayashi, M., Kato, H., Kakihana, M. Synthesis of titanium dioxide nanocrystals with controlled crystal- and micro-structures from titanium complexes. Nanomater. Nanotechnol. 3, 1-10 (2013).

30 Kobayashi, M., Tomita, K., Kakihana, M., Higuchi, T., Yoshino, R. The preparation method of peroxo-titanium compounds with low ammonium concentration. Japanese Patent No. 5008028 (2012).

31 Lamer, V. K., Dinegar, R. H. Theory, production and mechanism of formation of monodispersed hydrosols. J. Am. Chem. Soc. 72, 4847-4854 (1950).

32 Tomono, H., Nada, H., Zhu, F., Sakamoto, T., Nishimura, T., Kato, T. Effects of magnesium ions and water molecules on the structure of amorphous calcium carbonate: A molecular dynamics study. J. Phys. Chem. B 117, 14849-14856 (2013).

33 Zhu, F., Nishimura, T., Sakamoto, T., Tomono, H., Nada, H., Okumura, Y., Kikuchi, H., Kato, T. Tuning the stability of $\mathrm{CaCO}_{3}$ crystals with magnesium ions for the formation of aragonite thin films on organic polymer templates. Chem. Asian. J. 8, 3002-3009 (2013).

34 Zhang, Y., Wu, L., Zeng, Q., Zhi, J. An approach for controllable synthesis of differentphase titanium dioxide nanocomposites with peroxotitanium complex as precursor. J. Phys. Chem. C 112, 16457-16462 (2008).

35 Cheng, H., Ma, J., Zhao, Z., Qi, L. Hydrothermal preparation of uniform nanosize rutile and anatase particles. Chem. Mater. 7, 663-671 (1995).

36 Zheng, Y., Shi, E., Chen, Z., Li, W., Hu, X. Influence of solution concentration on the hydrothermal preparation of titania crystallites. J. Mater. Chem. 11, 1547-1551 (2001).

37 Gopal, M., Moberly Chan, W. J., De Jonghe, L. C. Room temperature synthesis of crystalline metal oxides. J. Mater Sci. 32, 6001-6008 (1997).

$38 \mathrm{Li}$, G., Gray, K. A. Preparation of mixed-phase titanium dioxide nanocomposites via solvothermal processing. Chem. Mater. 19, 1143-1146 (2007).

39 Testino, A., Bellobono, I. R., Buscaglia, C., Canevali, C., D’Arienzo, M., Polizzi, S., Scotti, R., Morazzoni, F. Optimizing the photocatalytic properties of hydrothermal $\mathrm{TiO}_{2}$ by the control of phase composition and particle morphology. A systematic approach. J. Am. Chem. Soc. 129, 3564-3575 (2007).

40 Wang, Y., Zhang, L., Deng, K., Chen, X., Zou, Z. Low temperature synthesis and photocatalytic activity of rutile $\mathrm{TiO}_{2}$ nanorod superstructure. J. Phys. Chem. C 111, 2709-2714 (2007).

$41 \mathrm{Li}$, J.-G., Ishigaki, T., Sun, X. Anatase, brookite, and rutile nanocrystals via redox reaction under mild hydrothermal conditions: Phase-selective synthesis and physicochemical properties. J. Phys. Chem. C 111, 4969-4976 (2007).

42 Zhang, Q., Gao, L. Preparation of oxide nanocrystals with tunable morphologies by the moderate hydrothermal method. Langmuir 19, 967-971 (2003).

43 Chemseddine, A., Moritz, T. Nanostructuring titania: Control over nanocrystal structure, size, shape, and organization. Eur. J. Inorg. Chem. 2, 235-245 (1999).

44 Reyes-Coronado, D., Rodrígues-Gattorno, G., Espinosa-Pesqueira, M., Cab, C., Coss, R., Oskam, G. Phase-pure $\mathrm{TiO}_{2}$ nanoparticles: anatase, brookite and rutile. Nanotechnol 19, 145605-145614 (2008).

45 Kinsinger, N. M., Wong, A., Li, D., Villalobos, F., Kisailus, D. Nucleation and crystal growth of nanocrystalline anatase and rutile phase $\mathrm{TiO}_{2}$ from a water-soluble precursor. Cryst. Growth Des. 10, 5254-5261 (2010).

46 Kobayashi, M., Petrykin, V., Kakihana, M., Tomita, K. Morphology control of rutile nanoparticles in a hydrothermal synthesis from water-soluble titanium complex aqueous solution. J. Ceram. Soc. Jpn 115, 835-839 (2007).

47 Kobayashi, M., Kato, H., Kakihana, M. Synthesis of spindle and square bipyramidshaped anatase-type titanium dioxide crystals by a solvothermal method using ethylenediamine. J. Ceram. Soc. Jpn 120, 494-499 (2012).

48 Kobayashi, M., Petrykin, V., Kakihana, M., Tomita, K. Hydrothermal synthesis and photocatalytic activity of Whisker-like rutile-type titanium dioxide. J. Am. Ceram. Soc. 92, S21-S26 (2009).

49 Ostwald, W. Studienüber die Bildung und Umwandlung fester Körper. Z. Phys. Chem. 22, 289-330 (1897).

50 Mendive, C. B., Bredow, T., Feldhoff, A., Blesa, M., Bahnemann, D. Adsorption of oxalate on rutile particles in aqueous solutions: a spectroscopic, electron-microscopic and theoretical study. Phys. Chem. Chem. Phys. 10, 1960-1974 (2008).

$51 \mathrm{Nada}, \mathrm{H}$. Difference in the conformation and dynamics of aspartic acid on the flat regions, step edges, and kinks of a calcite surface: A molecular dynamics study. J. Phys. Chem. C 118, 14335-14345 (2014).

52 Parker, R. A., Wasilik, J. H. Dielectric constant and dielectric loss of $\mathrm{TiO}_{2}$ (rutile) at low frequencies. Phys. Rev. 120, 1631-1637 (1960).

53 Parker, R. A. Static dielectric constant of rutile $\left(\mathrm{TiO}_{2}\right), 1.6-1060^{\circ} \mathrm{K}$. Phys. Rev. 124, 1719-1722 (1961).

54 Samara, G. A., Peercy, P. S. Pressure and temperature dependence of the static dielectric constants and Raman spectra of $\mathrm{TiO}_{2}$ (rutile). Phys. Rev. B 7, 1131-1148 (1973).

55 Patzke, G. R., Zhou, Y., Kontic, R., Conrad, F. Oxide nanomaterials: synthetic developments, mechanistic studies, and technological innovations. Angew. Chem., Int. Ed. 50, 826-859 (2011).

56 Gervais, F., Kress, W. Lattice dynamics of incipient ferroelectric rutile $\mathrm{TiO}_{2}$. Phys. Rev. B 28, 2962-2968 (1983).

57 Osada, M., Kobayashi, M., Kakihana, M. Enhanced dielectric response induced by controlled morphology in rutile $\mathrm{TiO}_{2}$ nanocrystals. J. Ceram. Soc. Jpn 121, 593-597 (2013).

58 Arlt, G., Hennings, D., de With, G. Dielectric properties of fine-grained barium titanate ceramics. J. Appl. Phys. 58, 1619-1625 (1985).

59 Grünebohm, A., Ederer, C., Entel, P. First-principles study of the influence of (110)oriented strain on the ferroelectric properties of rutile $\mathrm{TiO}_{2}$. Phys. Rev. B 84, 132105-132108 (2011). 